\title{
Effects of Cr underlayer and Pt buffer layer on the interfacial structure and magnetic characteristics of sputtered FePt films
}

\author{
An-Cheng Sun ${ }^{\mathrm{a}, \mathrm{b}}$, Jen-Hwa Hsu ${ }^{\mathrm{a}, \mathrm{b}, *}$, H.L. Huang ${ }^{\mathrm{a}, \mathrm{b}}$, P.C. Kuo ${ }^{\mathrm{a}, \mathrm{c}}$ \\ ${ }^{a}$ Center for Nanostorage Research, National Taiwan University, Taipei 106, Taiwan \\ ${ }^{\mathrm{b}}$ Department of Physics, National Taiwan University, Taipei 106, Taiwan \\ ${ }^{\mathrm{c}}$ Department of Materials Science and Engineering, National Taiwan University, Taipei 106, Taiwan
}

Available online 3 March 2006

\begin{abstract}
This work develops a new method for growing $\mathrm{L}_{0} \mathrm{FePt}\left(\begin{array}{ll}0 & 0\end{array}\right)$ thin film on a $\mathrm{Pt} / \mathrm{Cr}$ bilayer using an amorphous glass substrate. Semicoherent epitaxial growth was initiated from the $\operatorname{Cr}\left(\begin{array}{lll}0 & 0\end{array}\right)$ underlayer, continued through the $\operatorname{Pt}\left(\begin{array}{ll}0 & 0\end{array}\right)$ buffer layer, and extended into the $\mathrm{L1}_{0} \mathrm{FePt}\left(\begin{array}{ll}0 & 1\end{array}\right)$ magnetic layer. The squareness of the $\mathrm{L} 1_{0}$ FePt film in the presence of both a $\mathrm{Cr}$ underlayer and a Pt buffer layer was close to unity as the magnetic field was applied perpendicular to the film plane. The single $\mathrm{L}_{0} \mathrm{FePt}\left(\begin{array}{lll}1 & 1 & 1\end{array}\right)$ orientation was observed in the absence of a $\mathrm{Cr}$ underlayer. When a $\mathrm{Cr}$ underlayer is inserted, the preferred orientation switched from $\mathrm{L} 1_{0} \mathrm{FePt}(1111)$ to $\mathrm{L} 1_{0} \mathrm{FePt}(001)$ and the magnetic film exhibited perpendicular magnetic anisotropy. However, in the absence of an $\mathrm{Pt}$ intermediate layer, the $\mathrm{Cr}$ atoms diffused directly into the FePt magnetic layer and prevented the formation of the $\mathrm{L} 1_{0} \mathrm{FePt}\left(\begin{array}{ll}0 & 1\end{array}\right)$ preferred orientation. When a Pt buffer layer was introduced between the FePt and $\mathrm{Cr}$ underlayer, the $\mathrm{L} 1_{0} \mathrm{FePt}\left(\begin{array}{lll}0 & 0 & 1\end{array}\right)$ peak appeared. The thickness of the Pt buffer layer also substantially affected the magnetic properties and atomic arrangement at the $\mathrm{FePt} / \mathrm{Pt}$ and $\mathrm{Pt} / \mathrm{Cr}$ interfaces.
\end{abstract}

(C) 2006 Elsevier B.V. All rights reserved.

PACS: 81.15.C; 75.60.G; 75.70.K

Keywords: Magnetic perpendicular recording; FePt films; Pt buffer layer; $\mathrm{Cr}$ underlayer

\section{Introduction}

CoCr-base alloy thin films are widely employed in longitudinal magnetic recording media. The $M_{\mathrm{r}} t$ product of the media must be small to increase the linear density of magnetic recording, reduce the media noise, and improve the signal to noise ratio (SNR) [1]. ( $M_{\mathrm{r}} t$ is the product of remanent magnetization $M_{\mathrm{r}}$ and media thickness $t$.) The reduction in thickness of the media is always accompanied by shrinkage of the grain, causing thermally unstable magnetization at high linear density with a very large demagnetizing field $H_{\mathrm{d}}$. Antiferromagnetically coupled (AFC) magnetic recording media have been developed to improve the thermal stability of longitudinal media [2]. The

\footnotetext{
*Corresponding author. Department of Physics, National Taiwan University, Taipei 106, Taiwan. Tel.: + 886233665162 ; fax: +886233665892 .

E-mail address: jhhsu@phys.ntu.edu.tw (J.-H. Hsu).
}

$M_{\mathrm{r}} t$ of AFC media can be reduced without a decrease in the grain volume [1-3]. Therefore, a high-density recording of $200 \mathrm{G} / \mathrm{in}^{2}$ could be attained in AFC media. In future ultra-high-density magnetic recording applications, the AFC recording method will not be used because it yields a high $H_{\mathrm{d}}$ in the longitudinal direction and a minimal stable grain size that increases as the thickness of the media decreases. The perpendicular magnetic recording (PMR) method has been proposed to solve these problems of longitudinal media [4]. In PMR, the direction of magnetization is perpendicular to the film plane. If the surface area of the recording bit is reduced, the volume of the recording bit can still be maintained by increasing the medium thickness. Accordingly, the demagnetized field can be reduced to stabilize the magnetic moments [5].

FePt thin film with a tetragonal $\mathrm{L}_{0}$ structure and a ( 001 l) texture perpendicular to the film plane is a good candidate for perpendicular magnetic recording medium. The uniaxial magnetocrystalline anisotropic energy of the 
$\mathrm{L}_{0} \mathrm{FePt}$ phase is close to $10^{8} \mathrm{erg} / \mathrm{cm}^{3}$, which is 50 times greater than that of CoCr-based alloy thin films [6]. Unfortunately, the preferred orientation of the deposited $\mathrm{L} 1_{0} \mathrm{FePt}$ film is usually (1 11 ), so the easy axis is tilted $35^{\circ}$ from the film plane. In a perpendicular recording medium, the preferred orientation of the $\mathrm{L} 1_{0}$ FePt film must be with the $\left(\begin{array}{lll}0 & 0 & 1\end{array}\right)$ plane parallel to the film plane, making the easy crystallization axis [0 011$]$ perpendicular to the film plane.

Some methods for preparing an ordered $\mathrm{Ll}_{0} \mathrm{FePt}\left(\begin{array}{lll}0 & 0 & 1\end{array}\right)$ texture have been developed [7]. In these fabrication processes, $\mathrm{MgO}$ was extensively utilized to promote the preferred orientation with $\mathrm{FePt}\left(0_{0} 1\right)$ parallel to the film

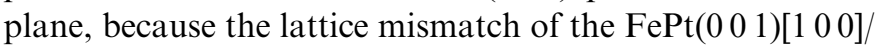

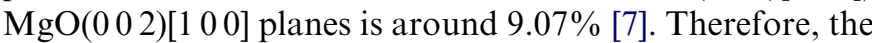
$\mathrm{MgO}(002)$ substrate or $\mathrm{MgO}(002)$ thin film can induce the $\mathrm{L} 1_{0} \mathrm{FePt}\left(\begin{array}{l}0 \\ 0\end{array}\right)$ texture. However, the cost of using an $\mathrm{MgO}(002)$ substrate is extremely high and the fabrication temperature of the $\mathrm{MgO}\left(0_{0} 2\right)$ thin film always exceeds $500{ }^{\circ} \mathrm{C}$ [8], limiting the adoption of $\mathrm{MgO}$ in the magnetic recording industry.

In this investigation, a $\mathrm{Pt}$ buffer layer and a $\mathrm{Cr}$ underlayer were used to induce the preferred orientation with $\mathrm{L1}_{0} \mathrm{FePt}(001)$ parallel to the film plane. This work examines the mechanism of formation and the interfacial characteristics of an ordered $\mathrm{L} 1_{0} \mathrm{FePt}\left(\begin{array}{lll}0 & 1\end{array}\right)$ film on a $\mathrm{Pt} / \mathrm{Cr}$ bilayer.

\section{Experimental}

All films were fabricated on preheated 7059 corning glass substrates by conventional DC magnetron sputtering in an ultra-high vacuum-sputtering chamber. The substrate was heated to $350^{\circ} \mathrm{C}$ to prepare the $\mathrm{Cr}$ underlayer and the $\mathrm{Pt}$ buffer layer. The deposition temperature of the FePt magnetic layer was set to $450{ }^{\circ} \mathrm{C}$ to overcome the order-disorder transformation energy barrier to yield the $\mathrm{L} 1_{0} \mathrm{FePt}$ phase [9]. The thickness of the films was measured by atomic force microscopy (AFM). The crystal structure and the cross-sectional microstructures of the films were investigated by X-ray diffraction (XRD) using $\mathrm{Cu}-\mathrm{K}_{\alpha}$ radiation and $300 \mathrm{keV}$ high-resolution transmission electron microscopy (HRTEM). The chemical composition of the magnetic FePt alloy layer was determined by energydispersive X-ray diffractometry (EDS), which revealed that the composition was $\mathrm{Fe}_{48} \mathrm{Pt}_{52}$. The element depth profiles of the films were investigated by Auger electron spectroscopy (AES). Magnetic properties were measured by a vibrating sample magnetometer (VSM) at room temperature.

\section{Results and discussion}

In our previous study [10], the magnetic FePt film with an ordered $\mathrm{L1}_{0} \mathrm{FePt}\left(0_{0} 1\right)$ preferred orientation was prepared on a $\mathrm{Pt} / \mathrm{Cr}$ bilayer using glass substrate. The thicknesses of the $\mathrm{Cr}$ underlayer and the $\mathrm{Pt}$ intermediate layer were 90 and $2 \mathrm{~nm}$, respectively. Fig. 1(a) displays an XRD pattern, which includes peaks from the $\operatorname{Cr}\left(\begin{array}{ll}0 & 02)\end{array}\right.$ reflection plane and $\left(\begin{array}{lll}0 & 0 & 1\end{array}\right)$ and $\left(\begin{array}{ll}0 & 0\end{array}\right)$ superlattice planes of the $\mathrm{L}_{0} \operatorname{FePt}(001)$ phase. The $\operatorname{FePt}(001)$ texture was preserved throughout the $\mathrm{L}_{0} \mathrm{FePt}$ layer by epitaxial growth from the $\operatorname{Cr}\left(0_{0} 2\right)$ underlayer. Additionally, the magnetic FePt film has been found to exhibit perpendicular magnetic anisotropy with an out-of-plane squareness $\left(M_{\mathrm{r}} /\right.$ $M_{\mathrm{s}}$ ) of about 1, as shown in Fig. 1(b). The saturation magnetization $\left(M_{\mathrm{s}}\right)$ could reach $700 \mathrm{emu} / \mathrm{cm}^{3}$. The easy axis of the film was perpendicular to the film plane.

The thickness of the $\mathrm{Cr}$ underlayer $d$ was varied from 0 to $110 \mathrm{~nm}$, while the film thickness of the FePt layer and Pt layer was fixed at 20 and $2 \mathrm{~nm}$, respectively, to explore the
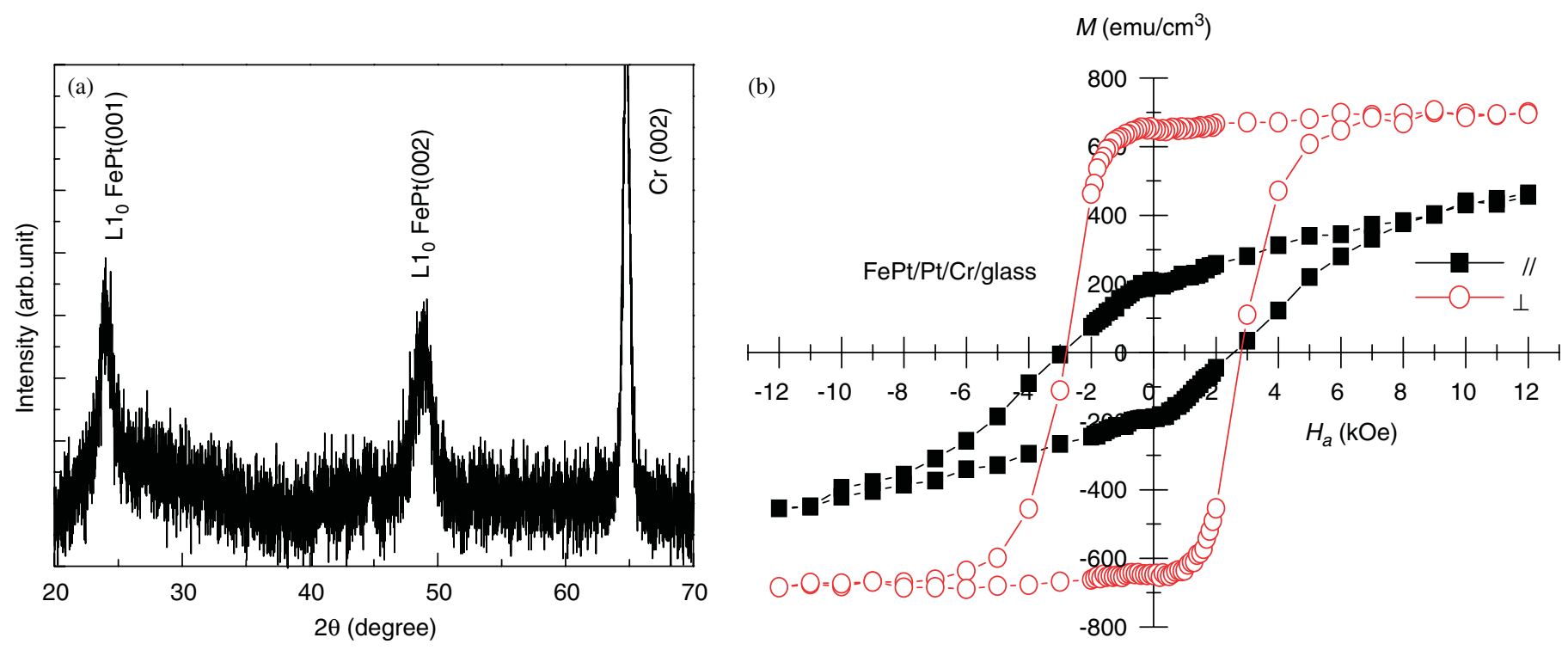

Fig. 1. (a) X-ray diffraction pattern and (b) $M-H$ loop of $\mathrm{FePt}(20 \mathrm{~nm}) / \operatorname{Pt}(2 \mathrm{~nm}) / \mathrm{Cr}(90 \mathrm{~nm})$ trilayer film. 


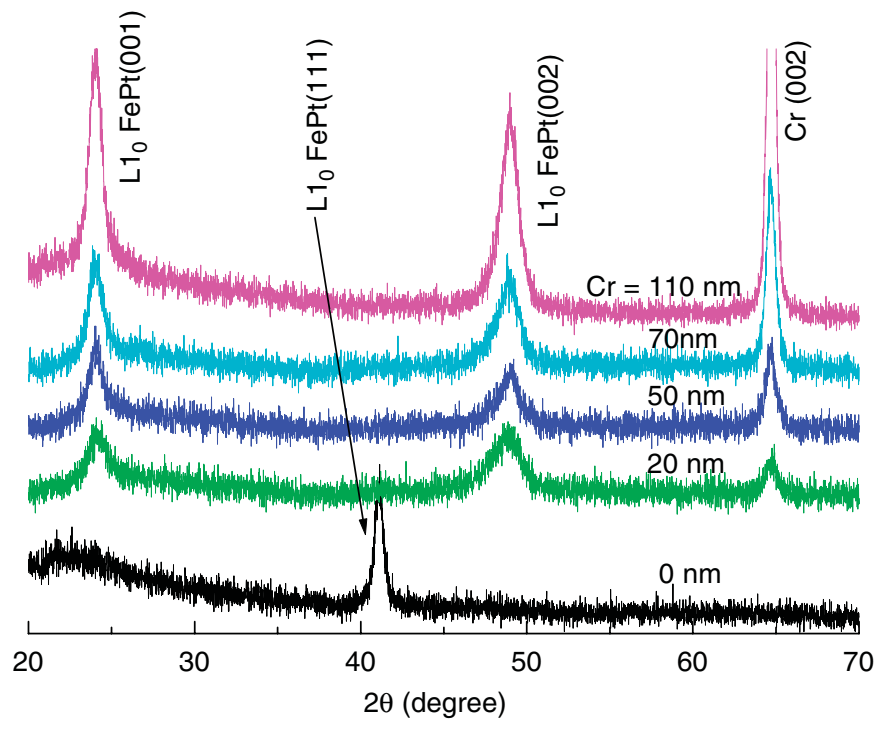

Fig. 2. The X-ray diffraction patterns of $\mathrm{FePt} / \mathrm{Pt} / \mathrm{Cr}$ trilayer films with various $\mathrm{Cr}$ underlayer thickness.

effect of the Cr underlayer. Fig. 2 depicts the XRD patterns of the $\mathrm{FePt} / \mathrm{Pt} / \mathrm{Cr}$ trilayers with $\mathrm{Cr}$ underlayers of various thicknesses. It shows that only a single $\mathrm{L}_{0} \operatorname{FePt}\left(\begin{array}{lll}1 & 1 & 1\end{array}\right)$ orientation was obtained when no $\mathrm{Cr}$ underlayer was present, because the lattice constant of the FCC Pt(llll 11 ) plane is close to that of the FCT FePt(lll 11$)$ plane. The FePt layer will be epitaxially grown along the $\mathrm{Pt}\left(\begin{array}{lll}1 & 1 & 1\end{array}\right)$ plane, yielding an FePt (1 111$)$-textured film. When the $\mathrm{Cr}$ underlayer was incorporated, $\mathrm{L} 1_{0} \operatorname{FePt}\left(\begin{array}{lll}0 & 0 & 1\end{array}\right)$ and $\operatorname{Cr}\left(\begin{array}{ll}0 & 0\end{array}\right)$ peaks were found instead. The $\operatorname{FePt}\left(\begin{array}{lll}1 & 1 & 1\end{array}\right)$ peak disappeared and the intensity of the $\operatorname{FePt}\left(\begin{array}{lll}0 & 0 & 1\end{array}\right)$ and $\operatorname{Cr}(002)$ peaks increased with the thickness of the $\mathrm{Cr}$ underlayer, indicating that the preferred orientation of the $\mathrm{L}_{0} \mathrm{FePt}$ grains switched from $\mathrm{FePt}\left(\begin{array}{lll}1 & 1 & 1\end{array}\right)$ to $\mathrm{FePt}\left(\begin{array}{lll}0 & 1 & 1\end{array}\right)$ as the $\mathrm{Cr}$ underlayer was added. The FePt( $\left(\begin{array}{lll}0 & 1\end{array}\right)$ texture was originated from the $\mathrm{Cr}\left(0_{0} 0_{2}\right)$ plane.

Fig. 3 plots the squareness $\left(M_{\mathrm{r}} / M_{\mathrm{s}}\right)$ and the out-of-plane coercivity $\left(H_{\mathrm{c} \perp}\right)$ of the $\mathrm{FePt} / \mathrm{Pt} / \mathrm{Cr}$ trilayer as a function of the $\mathrm{Cr}$ underlayer. In the absence of a $\mathrm{Cr}$ undrlayer, the inplane squareness $\left(S_{\|}\right)$was about 0.67 higher than the outof-plane squareness $\left(S_{\perp} \sim 0.59\right)$. When $d=20 \mathrm{~nm}, S_{\perp}$ increased dramatically to 0.92 , and $S_{\|}$dropped to 0.42 , suggesting that the perpendicular magnetic anisotropy dominated at $d=20 \mathrm{~nm}$. $S_{\perp}$ always exceeded 0.85 , even when the thickness of the Cr underlayer was increased to $110 \mathrm{~nm}$. $S_{\|}$fell as the thickness of the Cr underlayer increased; $S_{\|}$was largest (0.67) at $0 \mathrm{~nm}$ and it declined to about 0.16 at $d=110 \mathrm{~nm}$. The increase in the thickness of the $\mathrm{Cr}$ underlayer reinforced the perpendicular $\mathrm{FePt}\left(\begin{array}{lll}0 & 0 & 1\end{array}\right)$ orientation. The out-of-plane coercivity $\left(H_{\mathrm{c} \perp}\right)$ was also found to depend on the thickness of the $\mathrm{Cr}$ underlayer. $H_{\mathrm{c} \perp}$ was largest for the $\mathrm{FePt} / \mathrm{Pt}$ bilayer structure, with $d=0$. However, FePt/Pt bilayer did not exhibit perpendicular $\mathrm{FePt}\left(\begin{array}{lll}0 & 0 & 1\end{array}\right)$ anisotropy. Only the $\mathrm{FePt} / \mathrm{Pt} / \mathrm{Cr}$ trilayer system exhibited an $\operatorname{FePt}(001)$ texture with large $H_{\mathrm{c} \perp} . H_{\mathrm{c} \perp}$ increased from 2.8 to $3.6 \mathrm{kOe}$ as the thickness of the $\mathrm{Cr}$ underlayer increased from 20 to $70 \mathrm{~nm} . H_{\mathrm{c} \perp}$ decreases considerably as $d>70 \mathrm{~nm}$. These results were consistent with the results of a microstructure study [11]. The TEM plane view images of $\mathrm{FePt} / \mathrm{Pt} / \mathrm{Cr}$ trilayer films showed some black and white stripes in $\mathrm{FePt} / \mathrm{Pt} / \mathrm{Cr}$ films with a $90 \mathrm{~nm} \mathrm{Cr}$ underlayer, but not in such films with a $70 \mathrm{~nm} \mathrm{Cr}$ underlayer. The stripes might be caused by phase separation or local compositional inhomogeneity, which may reduce the magnetic anisotropy and out-of-plane coercivity.

The effects of the thickness of the Pt buffer layer on the microstructure and magnetic characteristics of the $\mathrm{FePt} / \mathrm{Pt} /$ $\mathrm{Cr}$ trilayer were also studied. Fig. 4 displays XRD patterns of the $\mathrm{FePt} / \mathrm{Pt} / \mathrm{Cr}$ trilayer with various $\mathrm{Pt}$ buffer layer thicknesses. The thicknesses of the FePt layer and the $\mathrm{Cr}$ underlayer were 20 and $90 \mathrm{~nm}$, respectively. Without a $\mathrm{Pt}$ buffer layer, the ordered $\operatorname{FePt}\left(\begin{array}{ll}0 & 0\end{array}\right)$ phase was difficult to

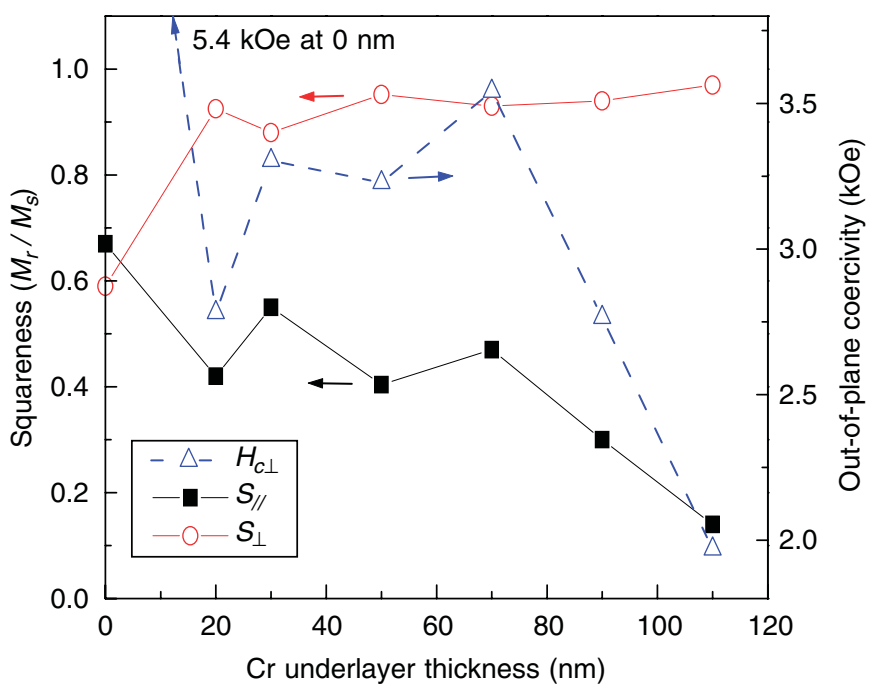

Fig. 3. The squareness $\left(M_{\mathrm{r}} / M_{\mathrm{s}}\right)$ and out-of-plane coercivity $\left(H_{\mathrm{c} \perp}\right)$ of $\mathrm{FePt} / \mathrm{Pt} / \mathrm{Cr}$ trilayer as a function of the $\mathrm{Cr}$ underlayer thickness.

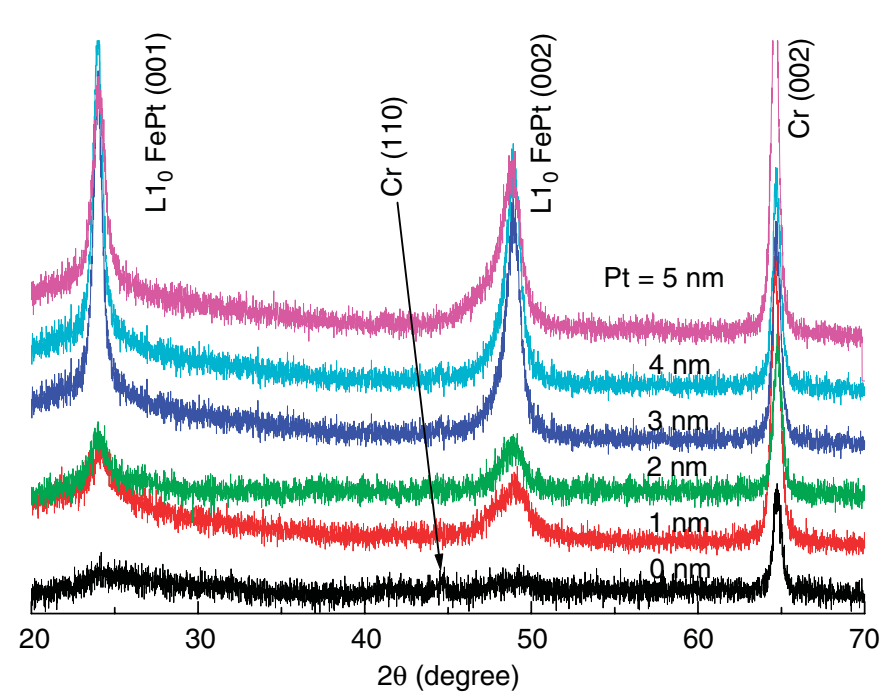

Fig. 4. The X-ray diffraction patterns of $\mathrm{FePt} / \mathrm{Pt} / \mathrm{Cr}$ trilayer with various Pt buffer layer thicknesses. 
identify. The intensity of the $\operatorname{Cr}(002)$ peak was low and the $\mathrm{Cr}\left(\begin{array}{lll}1 & 1 & 0)\end{array}\right)$ peak was present. This result was unexpected. The

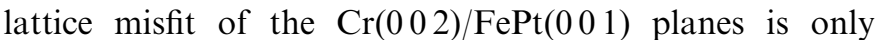
$5.8 \%$; so the ordered $\mathrm{FePt}(001)$ texture is expected to grow epitaxially with the crystal orientation $\operatorname{Cr}\left(\begin{array}{lll}0 & 0 & 2\end{array}\right)\left[\begin{array}{lll}1 & 1 & 0\end{array}\right] / /$ $\operatorname{FePt}\left(\begin{array}{lll}0 & 0 & 1\end{array}\right)\left[\begin{array}{ll}1 & 0\end{array}\right]$. However, this did not occur. The TEM cross-section image revealed that the epitaxial growth of $\mathrm{FePt}\left(\begin{array}{lll}0 & 0 & 1\end{array}\right)$ on the $\mathrm{Cr}\left(0_{0} 0_{2}\right) / \mathrm{FePt}\left(\begin{array}{lll}0 & 0 & 1\end{array}\right)$ interface was disrupted [10].

When the Pt layer was inserted between the magnetic FePt layer and the $\mathrm{Cr}$ underlayers, the $\mathrm{FePt}\left(\begin{array}{lll}0 & 0 & 1\end{array}\right)$ texture appeared, and the intensity of the $\operatorname{Cr}(002)$ peak was markedly increased. This result revealed that good epitaxial growth started from the $\mathrm{Cr}$ underlayer and extended to the $\mathrm{Pt}$ layer and the $\mathrm{FePt}$ magnetic layer. The lattice constant of $\mathrm{Pt}$ is $3.92 \AA$, which is between the $4.08 \AA$ of $\mathrm{Cr}\left(\begin{array}{ll}0 & 02\end{array}\right)$ and the $3.85 \AA$ of $\mathrm{FePt}(100)$. Hence, the intermediate Pt layer substantially reduces the strains at the $\mathrm{FePt} / \mathrm{Pt}$ and $\mathrm{Pt} / \mathrm{Cr}$ interfaces. Accordingly, the epitaxial growth can continue over a long distance. Consequently, the preferred orientation of the $\mathrm{L} 1_{0} \mathrm{FePt}$ phase was $\left(\begin{array}{ll}0 & 0\end{array}\right)$ and the film exhibited perpendicular magnetic anisotropy. In Fig. 4, the intensity of the $\operatorname{FePt}\left(\begin{array}{lll}0 & 0 & 1\end{array}\right)$ peak was highest when the thickness of the Pt layer was $3 \mathrm{~nm}$. Further increasing the thickness of the Pt layer reduced the height of the $\operatorname{FePt}\left(\begin{array}{lll}0 & 0 & 1\end{array}\right)$ peak. The $\mathrm{FePt}\left(\begin{array}{l}0 \\ 0\end{array}\right)$ peak became boarder as the Pt layer thickness increased above $3 \mathrm{~nm}$, perhaps because of the reduction of the order-disorder transformation of $\mathrm{FePt}$, and the associated decrease in the amount of $\mathrm{Ll}_{0} \mathrm{FePt}$ phase as the thickness of the Pt layer was increased. Interfaces of the $\mathrm{FePt} / \mathrm{Pt} / \mathrm{Cr}$ trilayer thin films with 2 and $4 \mathrm{~nm} \mathrm{Pt}$ buffer layers were examined to understand the drop of the ordering rate of FePt. Fig. 5 displays the TEM crosssectional images with Pt layers of thicknesses 2 and $4 \mathrm{~nm}$. The black and white arrows indicate the mismatch dislocation at the $\mathrm{Pt} / \mathrm{Cr}$ and $\mathrm{FePt} / \mathrm{Pt}$ interfaces, respectively. The mismatch dislocation can modulate the mismatch strain energy and cause the films to grow epitaxially and well at the interface [8]. When the thickness of the Pt layer was $2 \mathrm{~nm}$, many dislocations were present at the $\mathrm{FePt} / \mathrm{Pt} / \mathrm{Cr}$ interfaces, as shown in Fig. 5(a). However, when the thickness of the Pt buffer layer was increased to $4 \mathrm{~nm}$, mismatch dislocations at the interface were difficult
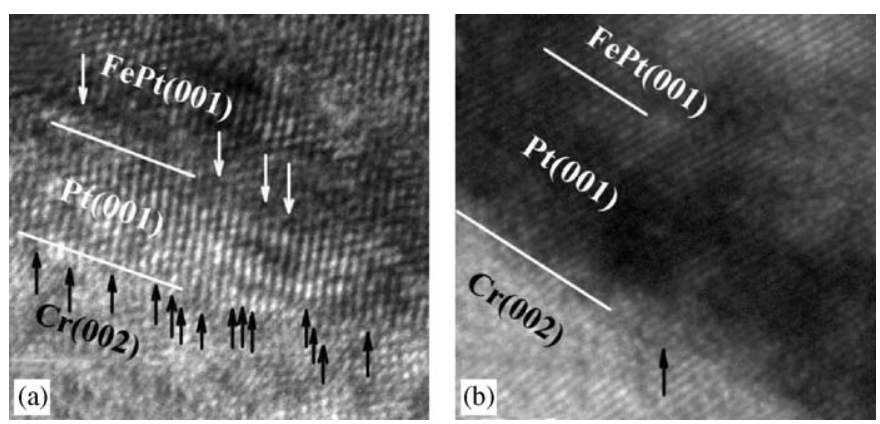

Fig. 5. TEM cross-sectional images with (a) $2 \mathrm{~nm}$ and (b) $4 \mathrm{~nm}$ Pt layer of $\mathrm{FePt} / \mathrm{Pt} / \mathrm{Cr}$ trilayer. to identify, as shown in Fig. 5(b). This result indicates that the lattice misfit strain energy is released gradually to yield a thicker Pt layer. The introduction of defects in the FePt thin film may promote the order-disorder transformation and reduce the ordering temperature $[11,12]$. The ordering temperature of a thicker Pt buffer layer $(4 \mathrm{~nm})$ was high because few mismatch dislocations were present. Therefore, a thicker Pt layer is associated with a less intense $\mathrm{L} 1_{0}$ $\mathrm{FePt}\left(0 \begin{array}{ll}0 & 1)\end{array}\right)$ peak in the XRD pattern (see Fig. 4).

Fig. 6 plots the squareness and out-of-plane $H_{\mathrm{c} \perp}$ versus the thickness of the Pt layer. $S_{\|}$exceeded $S_{\perp}$ when the Pt layer was thinner than $2 \mathrm{~nm}$, suggesting that the in-plane magnetic anisotropy was greater than the perpendicular anisotropy. $S_{\perp}$ was always higher than 0.9 and $S_{\|}$was smaller than 0.3 as the thickness of the Pt layer increased. Perpendicular magnetic anisotropy was realized if the $\mathrm{Pt}$ layer was thicker than $2 \mathrm{~nm}$. Without a Pt buffer layer, $H_{\mathrm{c} \perp}$ was only approximately $1.8 \mathrm{kOe}$. The AES element depth profile analysis revealed that the $\mathrm{Cr}$ atoms diffused directly into the FePt layer and distorted the FePt( $\left.\begin{array}{lll}0 & 0 & 1\end{array}\right)$ texture [10]. The saturation magnetization $\left(M_{\mathrm{s}}\right)$ of the $\mathrm{FePt} / \mathrm{Cr}$ bilayer was also reduced to be about $450 \mathrm{emu} / \mathrm{cm}^{3}$. A thin Pt buffer layer $(2 \mathrm{~nm})$ was found to suffice to impede the diffusion of the $\mathrm{Cr}$ atoms from the $\mathrm{Cr}$ underlayer into the $\mathrm{FePt}$ magnetic layer, yielding a better $\mathrm{FePt}\left(\begin{array}{ll}0 & 0\end{array}\right)$ orientation. The effect of the Pt buffer layer on the magnetic characteristics can be described as follows. $H_{\mathrm{c} \perp}$ increased from 1.8 to $3.0 \mathrm{kOe}$ as the Pt layer thickness increased from 0 to $3 \mathrm{~nm}$, and then declined to $2.5 \mathrm{kOe}$ as the thickness of the Pt layer was further increased from 3 to $5 \mathrm{~nm}$. The fall in $H_{\mathrm{c} \perp}$ as the $\mathrm{Pt}$ layer thickness increased above $3 \mathrm{~nm}$, and was related to the reduction in interfacial dislocations (Fig. 5(b)).

\section{Conclusion}

The epitaxial growth of $\mathrm{L}_{0} \mathrm{FePt}$ thin films on the $\mathrm{Pt} / \mathrm{Cr}$ bilayer with an amorphous glass substrate was studied.

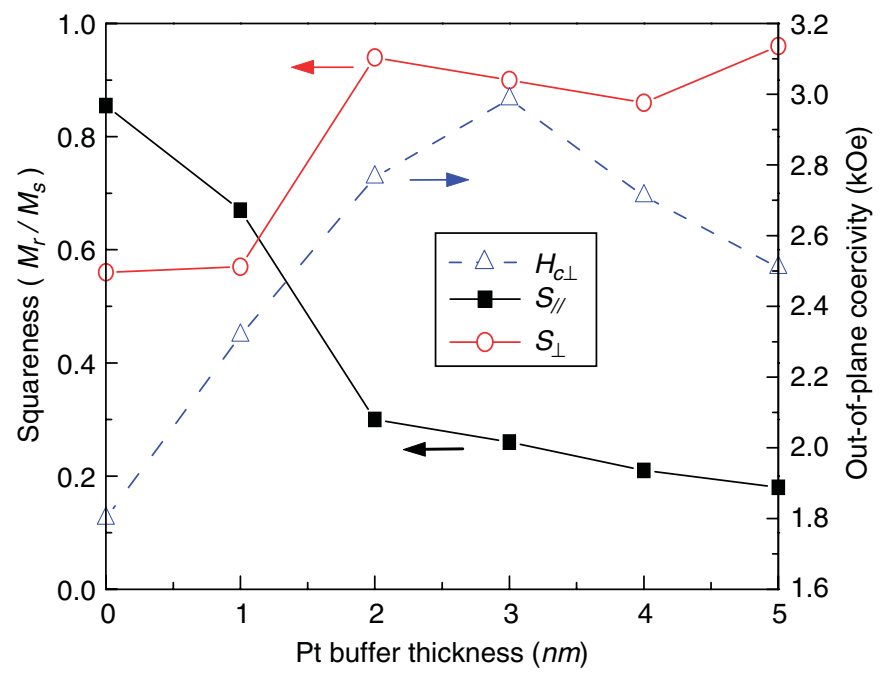

Fig. 6. The squareness $\left(M_{\mathrm{r}} / M_{\mathrm{s}}\right)$ and out-of-plane coercivity $\left(H_{\mathrm{c} \perp}\right)$ of $\mathrm{FePt} / \mathrm{Pt} / \mathrm{Cr}$ trilayer films as a function of the thickness of $\mathrm{Pt}$ buffer layer. 
Perpendicular magnetic anisotropy was present only with both a $\mathrm{Cr}$ underlayer and a Pt buffer. Epitaxial growth was initiated from the $\operatorname{Cr}(002)$ underlayer, continued through the Pt buffer layer, and extended into the $\mathrm{L} 1_{0} \operatorname{FePt}\left(\begin{array}{lll}0 & 1\end{array}\right)$ magnetic layer. Without a Pt buffer layer, the $\mathrm{Cr}$ atoms diffused directly into the FePt magnetic layer, eliminating the perpendicular magnetic anisotropy and preventing the epitaxial growth of the FePt magnetic layer. However, a thicker Pt buffer layer is responsible for the modulation of the mismatch strain energy, reducing the degree of interfacial dislocation at $\mathrm{FePt} / \mathrm{Pt} / \mathrm{Cr}$ interfaces. Hence, the order-disorder transformation energy of $\mathrm{FePt}$ cannot be lowered efficiently, resulting in the reduction of the perpendicular coercivity.

\section{Acknowledgements}

This work was supported by the Ministry of Economic Affairs of Taiwan, ROC, through Grant No. 93-EC-17-A08-S1-0006.

\section{References}

[1] E.N. Abarra, A. Inomata, H. Sato, I. Okamoto, Y. Mizoshita, Appl Phys. Lett. 77 (2000) 2581.

[2] Eric E. Fullerton, D.T. Margulies, M.E. Schabes, M. Carey, B. Gurney, A. Moser, M. Best, G. Zeltzer, K. Rubin, H. Rosen, M. Doerner, Appl. Phys. Lett. 77 (2000) 3806.

[3] M.E. Schabes, E.E. Fullerton, D.T. Margulies, IEEE Trans. Magn. 37 (2001) 1432.

[4] S. Iwasaki, Y. Nakamura, K. Ouchi, IEEE Trans. Magn. 15 (1979) 1456.

[5] Toshiyuki Suzuki, IEEE Trans. Magn. 20 (1984) 675.

[6] T. Shima, K. Takanashi, Y.K. Takahashi, K. Hono, Appl. Phys. Lett. 85 (2004) 2571.

[7] M.H. Hong, K. Hono, M. Watanabe, J. Appl. Phys. 84 (1998) 4403.

[8] K. Kang, Z.G. Zhang, T. Suzuki, C. Papusoi, J. Appl. Phys. 95 (2004) 7273.

[9] A.C. Sun, P.C. Kuo, S.C. Chen, C.Y. Chou, H.L. Huang, Jen-Hwa Hsu, J. Appl. Phys. 95 (2004) 7624.

[10] An-Cheng Sun, P.C. Kuo, Jen-Hwa Hsu, H.L. Huang, Jui-Ming Sun, J. Appl. Phys. 98 (2005) 076109.

[11] J.S. Chen, Yingfan Xu, J.P. Wang, J. Appl. Phys. 93 (2003) 1661.

[12] Yu-Nu Hsu, Sangki Jeong, David E. Laughlin, David N. Lambeth, J. Appl. Phys. 89 (2001) 7068. 